\title{
First steps to develop a sensor for a Tian-Calvet calorimeter with increased sensitivity
}

\author{
Franz Schubert ${ }^{1}$, Michael Gollner ${ }^{2}$, Jaroslaw Kita ${ }^{1}$, Florian Linseis $^{2}$, and Ralf Moos ${ }^{1}$ \\ ${ }^{1}$ Department of Functional Materials, University of Bayreuth, 95440 Bayreuth, Germany \\ ${ }^{2}$ LINSEIS Messgeräte GmbH, 95100 Selb, Germany
}

Correspondence to: Franz Schubert (functional.materials@uni-bayreuth.de)

Received: 11 February 2016 - Revised: 15 May 2016 - Accepted: 19 May 2016 - Published: 13 June 2016

\begin{abstract}
Initial steps to apply a ceramic multi-layer technique to build a new sensor for a Tian-Calvet calorimeter are presented in this contribution. The new sensor has a stacked design of ceramic sensor discs and insulating rings. The development was finite-element method (FEM) supported to design the sensor disc. In the next step, the function of the sensor disc was proven up to a temperature of $600^{\circ} \mathrm{C}$. Finally, the entire stack was tested at room temperature, delivering a resolution of $5 \mu \mathrm{W}$ and a maximum sensitivity of $8.5 \mu \mathrm{V} \mathrm{mW}^{-1}$. The time constant is strongly dependent on the mass of the cuvette. We show that the time constant of the sensor can be more exactly characterized when using a novel low temperature co-fired ceramic (LTCC) cuvette with a low mass and an integrated heater. Then, the time constant can be reduced to $T_{1 / e}=118 \mathrm{~s}$. The new sensor shows similar specifications as commercial devices and presents a good starting point for future high temperature applications.
\end{abstract}

\section{Introduction}

\subsection{The Tian-Calvet calorimeter and its special features}

Calorimeters are designed to measure heat produced by chemical or physical processes. Calorimetry data help, for example, one understand the temporal progress of chemical reactions, or to determine heat capacities or fusion enthalpies (Höhne et al., 2003). One group of well-established instruments is based on the so-called "Tian-Calvet" principle. Despite a large sample volume, this type of instrument shows remarkable sensitivity. It is generally used for high accuracy measurements of adsorption energies, chemical reaction heat, or ultra-accurate determinations of heat capacities (Becker and Gmehling, 2001), and many other applications that benefit from the large sample volumes (Auroux, 2013).

The Tian-Calvet calorimeter is based on the principle of differential measurements, meaning there are two identical cylindrical "sensors" 1 for heat, which are set up identically.

\footnotetext{
${ }^{1}$ The term "sensors", as it is used in the calorimetry community, may refer to a size of several centimeters or may encompass an entire device. It should not be mixed up with miniaturized devices that are called "sensors" in the metrology community.
}

One of these sensors measures the heat flow originating from the cuvette with a sample. The other sensor measures the heat flow originating from an inert reference, i.e., from an equal cuvette with a reference sample with an almost equal heat capacity to the sample. By subtracting both signals, the heat flow that is caused by the experimental setup can be eliminated from the signal. Thus, the heat flow caused by the processes in the sample can be measured with fewer interferences.

A drawback of those devices is the large time constant. It extends the time needed for titration experiments (Bäckman et al., 1994). Consequently, desmearing of the resulting signal is necessary (Lyon and Farris, 1990; Löblich, 1994).

In order to allow for a more accurate determination of chemical and physical properties, it is useful to increase the sensitivity of the sensor and to reduce its time constant. To understand the available opportunities to improve the calorimeter, we will first have a closer look into the existing setups. 


\subsection{Typical construction and possible approach for improvements}

The conventional heat-flux sensor consists of a thermopile that measures the temperature difference between the reference or the sample and the surrounding furnace, which has to be kept at a very uniform temperature. This is usually achieved by inserting the sensors into a metal block. It provides an identical temperature profile between the two sensors (Inaba et al., 1984; Kleppa, 2001; Mudd et al., 1982). The sensor itself has a total height of about $100 \mathrm{~mm}$ and consists of two thin concentrically arranged tubes. The outer one has a diameter of about $40 \mathrm{~mm}$ and is in thermally tight contact with the surrounding block. The inner tube is in contact with the cuvette with a diameter of $20 \mathrm{~mm}$ and a height of $70 \mathrm{~mm}$. An array of thermocouples (thermopile principle) is arranged between the tubes that measures the temperature difference between the inner and outer tube and thereby represents the difference in temperature between the sample and the furnace. Fabrication is most commonly performed by alternately welding two different types of wire together by hand and coupling the joints thermally to the tubes, yet insulating them electrically from the tubes. The space between the wires is filled with air. This process has not been changed much since the early concepts of Tian and Calvet (Calvet and Prat, 1956).

Possible methods to increase the sensitivity of the device comprise the minimization of the heat losses of the sensor by improving the thermal insulation. Thus, a chemical or physical process induces a higher temperature gradient, which is easier to detect. Furthermore, the number of thermocouples can be increased by increasing spatial density, which yields a higher voltage signal, although care has to be taken that the heat losses of the sensor are not adversely affected. One may also apply thermocouple materials with higher Seebeck coefficients.

All these points are difficult to address with the currently established manufacturing process as it leaves limited possibility for changes in design and limits the number of thermocouples because it restricts the maximum density of thermocouples. Moreover, materials with higher Seebeck coefficients are uncommon and expensive and are usually difficult to process into weldable wires.

In the past, different approaches have been developed to overcome these design limitations. One approach uses commercially available plate-shaped thermopiles for the sensor. Those sensors are built of block-shaped sintered ceramic thermoelectric materials with a high Seebeck coefficient. Therewith-built instruments exhibit a high sensitivity of up to $170 \mu \mathrm{V} \mathrm{mW}^{-1}$ (SETARAM Instrumentation, 2015) or even $763 \mu \mathrm{V} \mathrm{mW}^{-1}$ (Bäckman et al., 1994). However, to the best of our knowledge, no such device has been presented with an elevated operation temperature over $200^{\circ} \mathrm{C}$.

This may be due to the different shapes of sensor and sample container. Sample containers are cylindrical, which makes the thermal contact to the plate-shaped sensor difficult, requiring thermal coupling materials. This introduces a thermal mass and one more thermal contact into the system, and therefore increases the time constant of such a system.

Devices with a conventional wire design work at temperatures of up to $1500^{\circ} \mathrm{C}$ (Topor and Suponitskii, 1984). Sensitivities from $2.8 \mu \mathrm{V} \mathrm{mW}^{-1}$ (Setaram HT1000, operation temperature from room temperature to $1000^{\circ} \mathrm{C}$ ) (Razouk et al., 2013), through $15 \mu \mathrm{V} \mathrm{mW}^{-1}$ (proprietary instrument with an operating temperature between -73 and $317^{\circ} \mathrm{C}$ ) (Handy et al., 1993), to $30 \mu \mathrm{V} \mathrm{mW}{ }^{-1}$ (Setaram C80, operation temperature up to $300^{\circ} \mathrm{C}$ ) (SETARAM Instrumentation, 2016) can be reached. These data, however, indicate that for TianCalvet calorimeters the maximum operation temperature and the sensitivity behave inversely.

The goal of this study is to establish the baseline to develop a device that can work at temperatures above $300{ }^{\circ} \mathrm{C}$ with an increased sensitivity, compared to commercial devices.

To realize such an instrument, we kept a setup that is similar to the traditional system. Our main approach is to increase the number of thermocouples as well as to apply materials with higher Seebeck coefficients. To reach these goals, a low temperature co-fired ceramic (LTCC) technology is used to manufacture the sensor. In this technology, functional elements, in this case thermocouples, are screen printed as thick film structures on flexible ceramic green sheets. After firing, a ceramic structure with integrated functional elements is obtained. The technology is described in depth by Imanaka (2005) or Gongora-Rubio et al. (2001). Some recent developments in the sensor field are reviewed by Jurków et al. (2015). As a benefit, the thermal conductivity of LTCC is lower than that of metal and the thickness of the conducting metal can be decreased, which is beneficial for thermal insulation of the sensor.

The disadvantage of this technique is the typical ceramic brittleness, not allowing for a monolithic structure of the entire sensor. Therefore, the sensor was built by alternately stacking two small main components - the sensing component as a series connection of circular sensor elements, each having a ring of a radial thermopile around it and rings of insulation material filling the space between the sensor discs, therefore allowing one to form the full sensor volume. Figure 1a shows the conventional design with the series connection of the wire thermocouples and Fig. 1b depicts the new concept.

In order to prove the validity of the approach, the layout of the sensor disc was designed and it was optimized using a FEM model. After that, a furnace was built in which a single sensor disc was tested at elevated temperatures. At the end of this initial study, the complete sensor was tested at room temperature. 


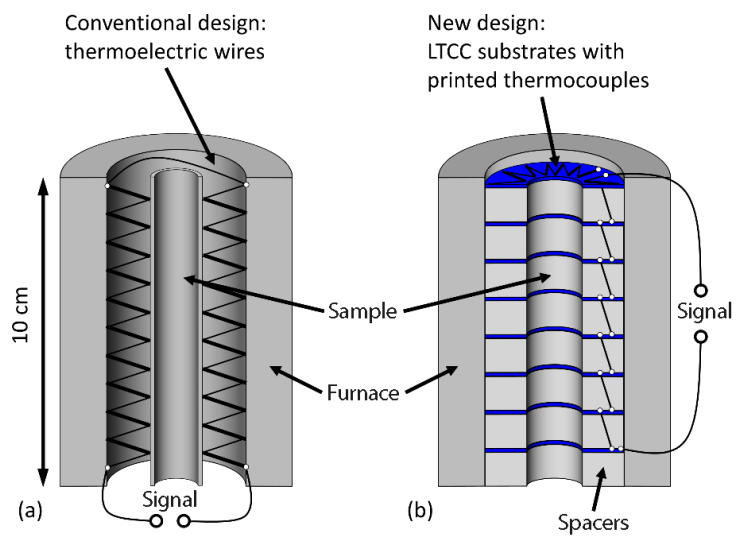

Figure 1. Conventional design of the sensor with thermoelectric wires (a) and the new design (b) with LTCC substrates with printed thermocouples.

\section{Experimental}

\subsection{Initial design, FEM modeling, and fabrication of the sensor disc and the sensor}

For the body of the sensor disc, DuPont 951P2 LTCC tape was used (Imanaka, 2005; Kita and Moos, 2008). The thermal conductivity of LTCC is much lower than that of alumina (a.k.a. aluminum oxide), which is typically used in thick film technology (Kita et al., 2015). This increases the sensitivity. Additionally, structuring of green LTCC sheets is easier than structuring of sintered ceramics, which has been reported for complex structures for applications with similar demands (Kita et al., 2005; Missal et al., 2011). For the thermocouples, the choice of materials is very limited. In the past years, the application of silver, silver-palladium, and nickel inks have been reported (Markowski et al., 2007). As shown in previous works (Wiegärtner et al., 2015), thermocouples of gold $(\mathrm{Au})$ and platinum $(\mathrm{Pt})$ have led to good results, too. Therefore, for the here-reported initial tests, an Au-Pt combination was selected, using the TC 7102 thick-film paste (Heraeus) for gold and the LPA 88-11 S thick-film paste (Heraeus) for platinum elements.

Prior to printing, four sheets of LTCC tape were laminated for $10 \mathrm{~min}$ at $21 \mathrm{MPa}$ and $70^{\circ} \mathrm{C}$. The thermocouples were made of screen-printed gold and platinum layers with a thickness of ca. $7 \mu \mathrm{m}$ and a width of $200 \mu \mathrm{m}$. The final form of the sensor was cut out with a frequency tripled Nd:YAG laser and sintered for $30 \mathrm{~min}$ at $850^{\circ} \mathrm{C}$.

The design of the sensor disc was supported by FEM analyses. The goal was to find how cutouts in the LTCC substrate increase the sensitivity of the sensor. Material data for gold and platinum were taken from Touloukian et al. (1970), data for LTCC were used as determined by Kita et al. (2015). A constant power of $P=1 \mathrm{~W}$ was assumed in the area of the sample and the temperature at the outer surface was considered as constant at an intermediate operation temperature of

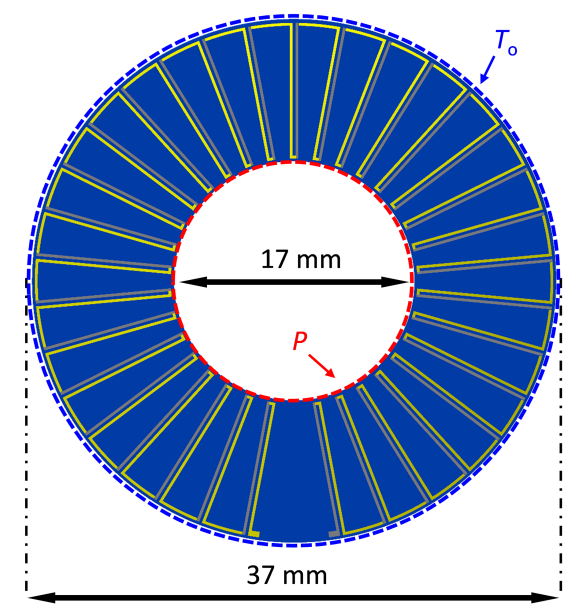

Figure 2. Initial design of a sensor disc with boundary conditions for modeling with the power of the sample $P$ and external temperature of the furnace $T_{\mathrm{O}}$.

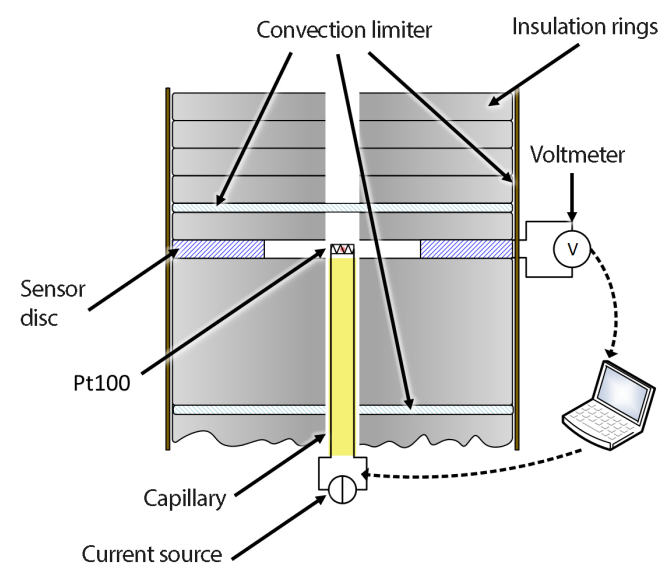

Figure 3. Sketch of the inner part of the initial setup to test the sensor discs.

$T_{\mathrm{o}}=400^{\circ} \mathrm{C}$. From the temperature increase in the area of the sample, a thermal resistance was calculated. For modeling, a sensor disc was designed with 33 thermocouples to provide some room for connection. The thermocouples show a radial pattern with some space between the individual paths, as can be seen from Fig. 2.

The complete sensor was produced by alternately stacking eight sensor discs and eight rings made of machined porous aluminosilicate. Gold wires between the discs were sintered to the contact pads by means of gold paste to connect the discs electrically.

\subsection{Tests of the single sensor disc and the complete sensor}

Initial tests were conducted to prove the basic function of the sensor discs up to elevated temperatures. Figure 3 sketches the measurement setup to test one single disc. The sensor 
disc was positioned in the middle of the insulation material. The inner area of the disc was heated by a Pt100 element with a computer-controlled current source. The whole unit was inserted into a custom-built test furnace with a maximum temperature of $600^{\circ} \mathrm{C}$. The sensor voltages for different heating power levels were measured by a custom-built voltage measurement unit. Experiments were conducted up to $600^{\circ} \mathrm{C}$ in steps of $200^{\circ} \mathrm{C}$. The leads were guided through a capillary with a thickness of $4 \mathrm{~mm}$ to ensure the correct positioning of the Pt100 heating resistor. The area around disc and heater was shielded by a convection limiter made of two alumina rings and a metal hull.

Figure 4 shows a sketch of the two stacks as they are implemented into a test setup. The stacks were inserted into an isothermal copper block with two bores to ensure a uniform temperature distribution around the stacks. Both stacks were interconnected in a differential method to eliminate additive noise that occurs in both sensors symmetrically. To achieve the differential setup, the signal $U_{\mathrm{S}}$ of the sensors was measured between the positive poles of each stack. In this case, the positive pole is defined as the pole where a voltmeter indicates a positive voltage if a cuvette emits heat. The negatives of each sensor are connected to form the differential setup. To prevent heat losses through the top and bottom surfaces, additional insulation was applied. The isothermal block was surrounded by insulation material to ensure minimum disturbances from the surrounding air. In order to test the sensitivity of the sensors, no defined reaction was used but instead a caloric calibration procedure, which is a widely recommended standard in literature, was applied (Barale et al., 2015; Sarge et al., 1994). A Pt1000 was placed freely suspended in the middle of a metal cuvette with mass of $25 \mathrm{~g}$ and supplied with different currents by a computer-controlled current source. Through measuring the current $I$ and the voltage $U$, the generated heat $P=U \times I$ could be accurately determined. In a typical test program, different power steps from $5 \times 10^{-7}$ to $10^{-3} \mathrm{~W}$ were impressed. All leads were made of gold $(\varnothing 100 \mu \mathrm{m})$ to limit thermoelectric noise from wiring.

As it was noticed that the time constant of the system depends strongly on the mass of the cuvette, a lighter cuvette, made of wrapped and laminated LTCC tapes with an integral heater, was produced according to Brandenburg et al. (2013) for final experiments (see at the end).

\section{Results and discussion}

\subsection{Initial design, FEM modeling, and fabrication of the sensor disc and the sensor}

Figure 5 shows modeling results of two discs with 33 thermocouples with and without implemented cutouts. As expected, the temperature on the disc drops from the inside to the outside. The resulting temperature gradient is $35.2 \mathrm{~K}$ for the disc without cutouts and $68.5 \mathrm{~K}$ for the disc with cutouts.

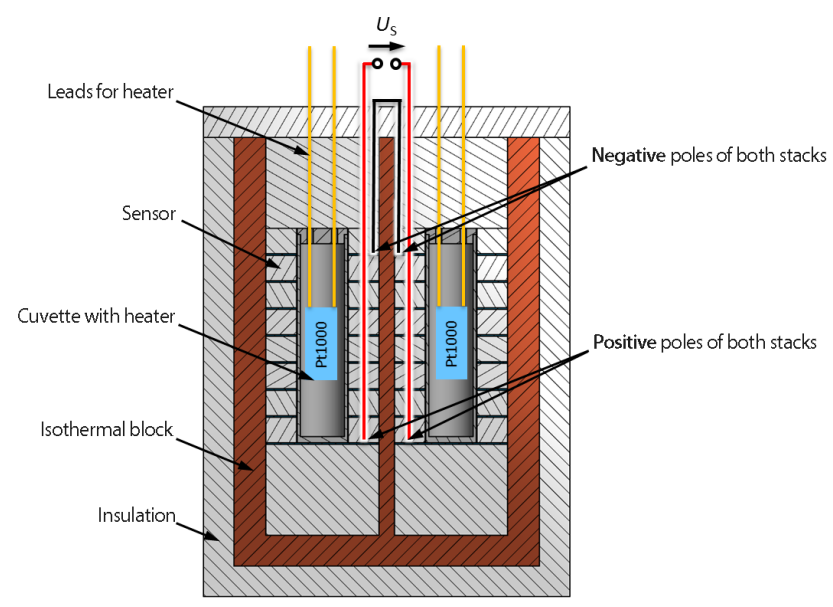

Figure 4. Insulated isothermal copper block with sensors and cuvettes for testing. $U_{\mathrm{S}}$ denotes the sensor signal.
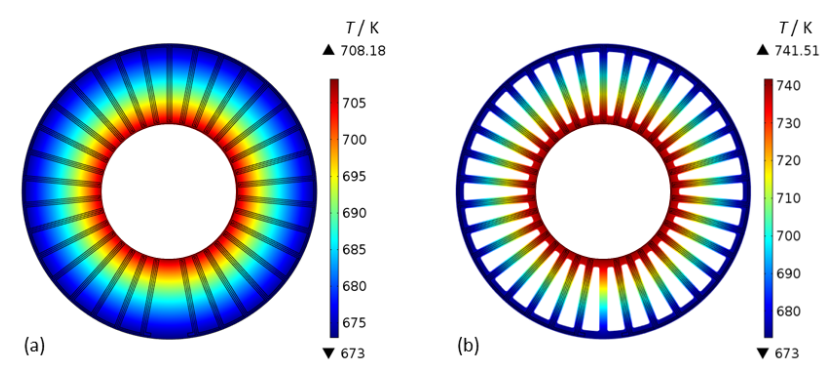

Figure 5. Result for the modeling of the temperature gradient that results when a power of $P=1 \mathrm{~W}$ is applied in the middle of the sensor disc and the outer surface is kept at $400^{\circ} \mathrm{C}$. The comparison between the temperatures of the sensor discs without (a) and with cutouts (b) between the 33 thermocouples shows the positive effect of the cutouts.

The thermal resistance is proportional and yields in 35.2 and $68.5 \mathrm{~K} \mathrm{~W}^{-1}$, i.e., the cutouts almost double the thermal resistances. Therefore, cutouts were inserted in the manufactured sensor discs.

Figure 6 shows one sensor disc in its final layout with 34 thermocouples at different production stages. Experiments for contacting the sensor disc had shown that one additional thermocouple fits on the sensor disc. At the bottom of the structure, three pads can be seen: two of them are needed to connect different discs in series, and the additional pad serves to connect a single thermocouple to measure the absolute temperature of the sample. Difference in roundness from the vertical to the horizontal diameter were less than $0.1 \mathrm{~mm}$ in the sintered structure.

Figure 7 is a photograph showing a side view of a finished sensor with eight sensor discs and a total of 272 thermocouples. The sensor discs fit well between the insulation layers. The height of the sensor is $70 \mathrm{~mm}$ in total. 


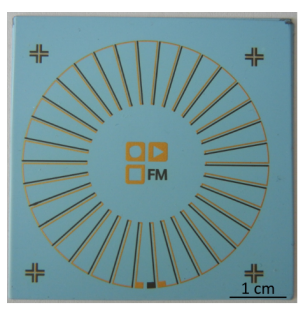

(a)

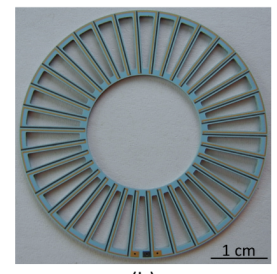

(b)

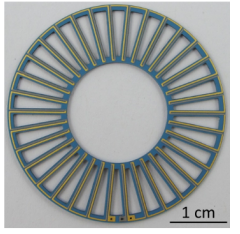

(c)
Figure 6. Sensor disc with 34 thermocouples in different production stages: after screen-printing (a), after laser patterning (b) and the finalized disc after sintering (c).

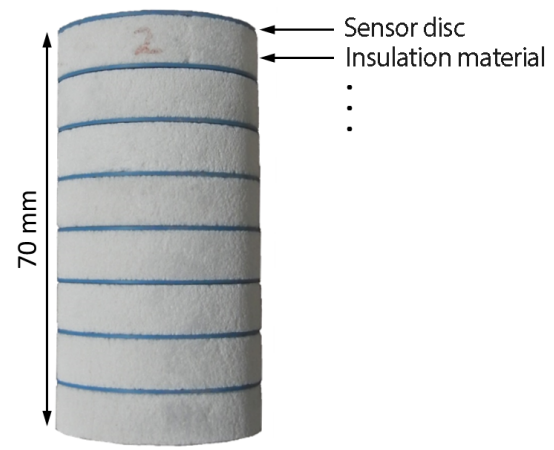

Figure 7. Finalized sensor stack with eight sensor discs.

\subsection{Test of a single disc}

Initial tests demonstrated the proper function of the sensor discs in the tested temperature range (Fig. 8). For all temperatures, power levels of $0.1 \mathrm{~W}$ could be detected. As the Seebeck-coefficient of gold and platinum nearly triples when the temperature increases from room temperature to $600{ }^{\circ} \mathrm{C}$ (Gotoh et al., 1991), at $600^{\circ} \mathrm{C}$ even the power of $0.01 \mathrm{~W}$ induces a clearly detectable signal. This measurement series proved the function of the sensor disc up to $600^{\circ} \mathrm{C}$. No negative effect on mechanical stability was observed.

The two main artifacts (high offset during heating and the temperature dependency of the baseline) can be fully attributed to the setup, since at this stage no differential setup was used. The offset is caused by the thermal inertia of the sensor, which induces a temperature gradient that is detected by the sensor disc. The reason for the baseline shift to more negative values as the temperature rises is the increasing heat flow through the leads for the Pt100 and the alumina used for the capillary. Heat flow through the capillary and the wires is proportional to the difference in temperature from the inner temperature of the disc to room temperature. An increase of the temperature for the measurement also increases heat losses through the capillary. Hence, it decreases the temperature of the sample and increases the magnitude of the baseline shift.
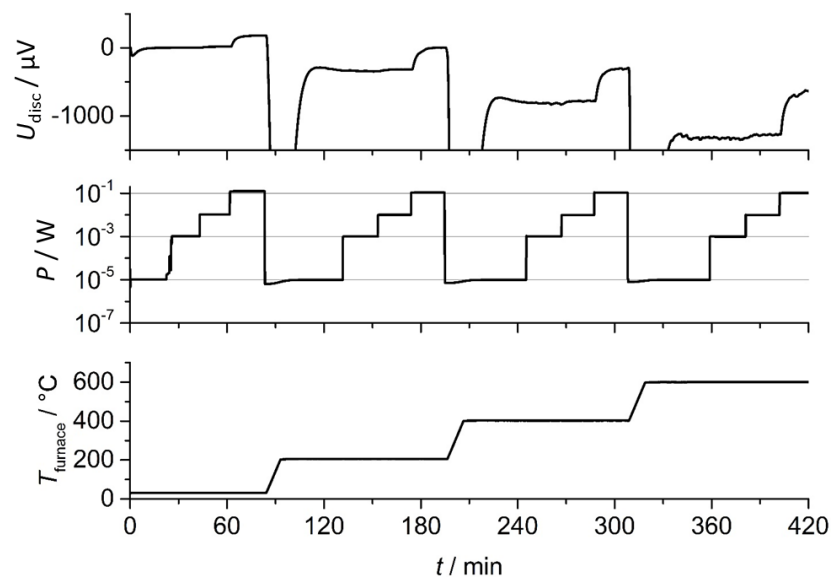

Figure 8. Results of initial tests for a sensor disc at various temperatures. The resulting voltage in the sensor disc $\left(U_{\text {disc }}\right)$ was measured dependent on the applied power $P$ and the furnace temperature $T_{\text {furnace }}$.

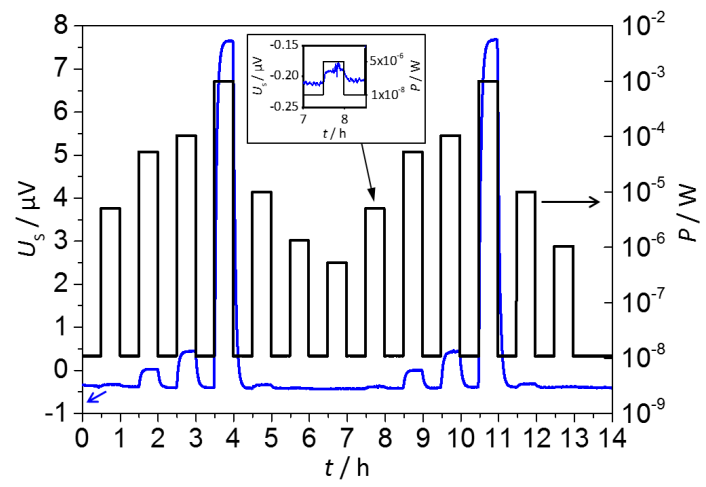

Figure 9. Results for initial tests of the stack. Different power steps $P$ were impressed to the cuvette and the resulting signal $U_{\mathrm{S}}$ was measured.

Since the principle had been proven, the development could proceed to the next step, the test of a fully assembled sensor, the "entire sensor".

\subsection{Test of the entire sensor}

Figure 9 shows how an entire sensor stack responds to applied power pulses. The obtained voltage signal $U_{\mathrm{s}}$ was smoothed by a moving average over 50 values. The sampling rate was about $1 \mathrm{~Hz}$. It can be seen that the signal of the sensor follows the power impressed into the testing cuvette with a minimum detectable power level of approximately $5 \mu \mathrm{W}$. The voltage signal is stable within $\pm 0.1 \mu \mathrm{V}$ during the whole duration of the experiment $(14 \mathrm{~h})$. The total noise of the signal is about $25 \mathrm{nV}_{\mathrm{rms}}$. A time constant of $T_{1 / e}=181 \mathrm{~s}$ can be calculated from the raw data of the signal and is a typical value for Tian-Calvet-type instruments. Data from literature for commercial devices vary from 280 to 360 s (Barale et al., 


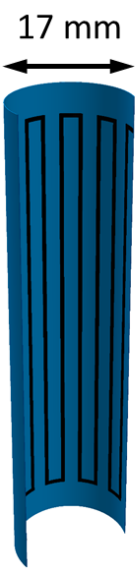

(a)

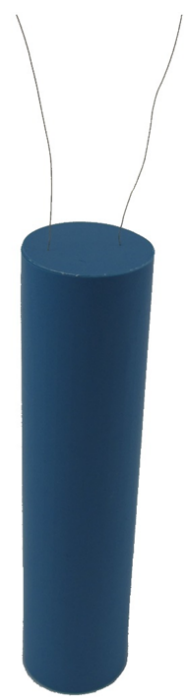

(b)
Figure 10. The drawing (a) shows half of the tube with integrated heating elements after sintering. The picture of the finished cuvette (b) was taken after the heating elements were contacted with wires and the open ends of the tube closed with lids.

2015). However, the time constants do not only depend on the sensor itself but are also strongly dependent on the used calibration device and its mass. It was assumed that the relatively high cuvette mass of $25 \mathrm{~g}$ and the poor thermal connection of the freely suspended Pt1000 affect the results.

In literature, a thermal RC circuit is often assumed to describe the calorimeter (Calvet and Prat, 1956; Schönborn, 1983). If the power of the sample follows a step function, then the sensor signal follows Eqs. (1) and (2).

$U=P \times s \times\left(1-e^{\frac{t}{\tau}}\right)$

$\tau=R \times C$

In Eqs. (1) and (2), $P$ is the power of the sample, and $s$ is sensitivity of the sensor, $t$ denotes the time, $\tau$ the time constant of the sensor with sample, and $R$ is the thermal resistance between the sample and the inner surface of the furnace. $C$ stands for the heat capacity of the sensor and the sample. This model is very limited and more complex mathematical multi-body theories for calorimeters have been developed (Tanaka, 1989; Zielenkiewicz et al., 1985; Saito et al., 1986). However, as they are not applied frequently in practice (Zielenkiewicz, 2004), we will stay with the simple model for explaining the time constant of the sensor.

According to the model, if the mass of the cuvette is increased, the heat capacity $C$ increases and the time constant $\tau$ does, too. The freely suspended Pt1000 in the cuvette increases the thermal resistance $R$ of the system, which also has a detrimental effect on the time constant. For determining the true time constant of the sensor, the mass of the cuvette has to be reduced and the heating element has to be incorporated as close to the sensor as possible. For that reason, an LTCC cuvette with an integrated heater seems suitable.

Figure 10 shows the LTCC cuvette. Length and width are adjusted to the inner dimensions of the sensor. The heating elements are made of platinum conductor tracks that are located on the walls of the tube. Their pattern was calculated in a way that the entire perimeter of the cuvette is covered. The resulting resistance after firing was about $650 \Omega$. Figure 10a shows a sketch of half a tube with the intern heater pattern to illustrate the design. The real cuvette (Fig. 10b) has an additional layer of LTCC on the inside of the tube to protect the heater structure; therefore, the walls have a thickness of $250 \mu \mathrm{m}$. After the tube is contacted via gold wires and closed with lids (also made of LTCC), the total mass of the cuvette is about $3 \mathrm{~g}$, which is a reduction of $88 \%$ compared to the steel cuvette. According to measurements at LINSEIS Messgeräte GmbH, DuPont 951 has a specific heat capacity of $0.73 \mathrm{~J}(\mathrm{~g} \times \mathrm{K})^{-1}$, which results in a heat capacity of the cuvette of $2.19 \mathrm{~J} \mathrm{~K}^{-1}$. The material of the steel cuvette (V2A) has a mass of $25 \mathrm{~g}$ and a specific heat capacity of $0.47 \mathrm{~J}(\mathrm{~g} \times \mathrm{K})^{-1}$ (VDI, 2013). Therefore, the resulting heat capacity is $11.75 \mathrm{~J} \mathrm{~K}^{-1}$; i.e. the LTCC cuvette reduces heat capacity by $81 \%$.

In Fig. 11, the signal of the metal and LTCC cuvettes are compared. For better comparability, both signals were compensated for offset and smoothed by a moving average of 50 values. With both cuvettes, a similar signal level was reached; however, with the LTCC cuvette, the response time is much faster. Since the heating resistor is now integrated into the wall of the cuvette, a considerable part of the thermal transfer resistance is reduced. Consequently, the delay drops from 11 down to $1 \mathrm{~s}$ from the point in time when power was applied to the resistor to the first increase of the sensor response. The time constant decreases to a value of $118 \mathrm{~s}$, which is a reduction of $35 \%$. It is assumed that this value is very close to the time constant of the ideal sensor without sample.

Figure 12 shows the sensitivity $(s)$ of the sensor as a function of the power $(P)$ that was impressed into the cuvettes. $s$ was calculated according to Eq. (3).

$s=\frac{U_{\mathrm{ss}}}{P}$,

where $U_{\text {ss }}$ stands for the voltage in steady state and $P$ denotes the released power of the cuvette.

For the metal cuvette, the measured sensitivity is $(7.7 \pm$ 0.7) $\mu \mathrm{V} \mathrm{mW}^{-1}$. It is not constant over the whole power range, but decreases when the power is lower than $5 \times 10^{-5} \mathrm{~W}$. It is assumed that this is an effect of the cuvette rather than of the sensor. The reason is based on the special conditions of the heat transfer from the freely suspended Pt1000 element. There are two paths for heat transfer away from the Pt1000 heater, one by convection through the surrounding air in the 


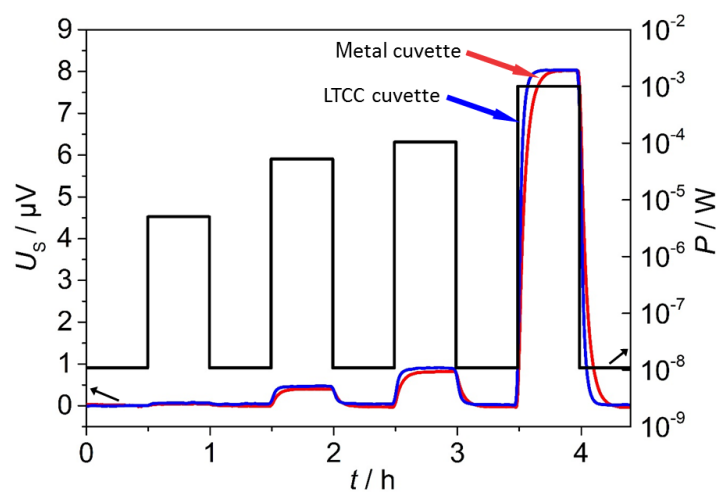

Figure 11. Impressed power $P$ into the cuvettes and sensor signal $U_{\mathrm{S}}$ for the metal cuvette and for the LTCC cuvette.

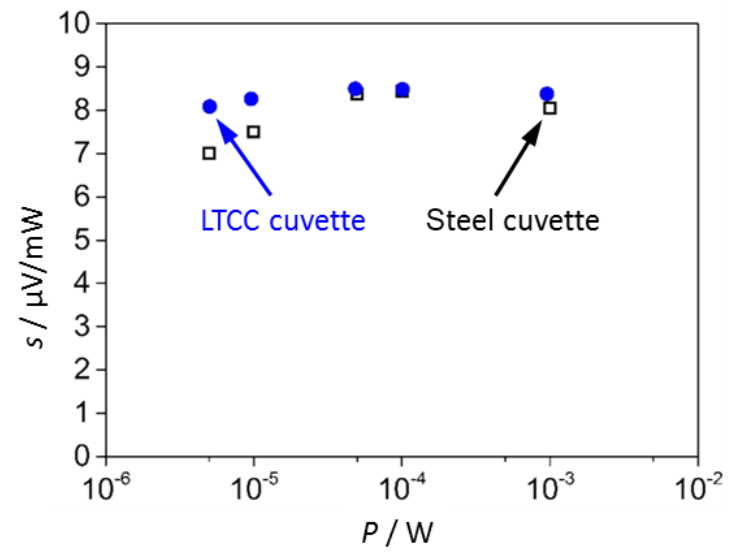

Figure 12. Sensitivity $s$ of the sensor for a metal cuvette and an LTCC cuvette when different power levels $P$ are impressed. The steel cuvette is indicated by a $\square$ symbol, blue dots denote the LTCC cuvette.

cuvette and one by conduction through the suspending wires. Conduction is proportional to the impressed power. The natural convection, in contrast, becomes nonlinear as the PT1000 has a small volume and its temperature varies strongly over the 3 decades of impressed power. At lower power levels, natural convection is low and a bigger proportion of heat is lost through the wiring. Thus, the measured sensitivity decreases. This is not the case with the LTCC cuvette, as there is no natural convection involved in heat transfer. Therefore, the measured sensitivity is more constant to the impressed power. Additionally, the power is distributed over a bigger volume and the temperature rise of the LTCC cuvette is smaller than that of the Pt1000. Therefore, heat conduction through the wiring is reduced, resulting in an increased measured sensitivity to a level of $(8.3 \pm 0.2) \mu \mathrm{V} \mathrm{mW}^{-1}$.

\section{Conclusion and outlook}

Initial steps to develop a new Tian-Calvet calorimeter for operation at elevated temperatures is reported in this study. The initial model showed that reducing the thermal conduction over the sensor disc increases sensitivity. Therefore, a design with 34 thermocouples (gold/platinum) with cutouts between the individual paths was chosen. Experimental data of a single sensor disc up to $600^{\circ} \mathrm{C}$ validate the approach, as the sensor exhibited a stable signal and mechanical integrity. The sensitivity of the disc increased with temperature, which is an effect of the increase of the Seebeck coefficient with temperature. The complete sensor stack with 8 discs of 34 thermocouples each was built and measurements were conducted at room temperature in a differential setup. The high mass of a metal testing cuvette increased the time constant of the sensor and the freely suspended heating resistor, which was used in the tests to apply a defined power, made the sensitivity dependent on the impressed power. With a novel LTCC cuvette with an integrated heater, this dependency could be counteracted. The time constant of the system decreased as well. The final setup shows a maximum sensitivity of $8.5 \mu \mathrm{VmW}^{-1}$ and a time constant of $T_{1 / e}=118 \mathrm{~s}$ with a resolution of $5 \mu \mathrm{W}$. Sensitivity and time constant of the sensor stack are on the same order of magnitude as for commercial sensors. Moreover, the sensitivity strongly increases with temperature.

This work has thereby successfully proven the concept of applying LTCC technology to a Tian-Calvet calorimeter.

Future work will focus on elevated temperature concepts and on increasing the sensitivity of the system, i.e., by increasing the number of sensor discs and/or the number of thermocouples on the sensor. Another possibility is to utilize other thermoelectric materials such as nickel, which has been successfully applied to LTCC (Markowski et al., 2007). This may increase the sensitivity by another factor of 3.3.

Acknowledgements. The presented results have been achieved in the scope of the project MST-1304-0003//BAY182/002 "HighResolution Calorimetric Sensor", which is funded by the Bavarian Ministry of Economic Affairs and Media, Energy and Technology, VDI/VDE-IT Microsystems Technology Program.

Edited by: Winfried Vonau

Reviewed by: two anonymous referees

\section{References}

Auroux, A.: Calorimetry and Thermal Methods in Catalysis, 154, Springer, Berlin, Heidelberg, 2013.

Bäckman, P., Bastos, M., Hallén, D., Lönnbro, P., and Wadsö, I.: Heat conduction calorimeters: time constants, sensitivity and fast titration experiments, J. Biochem. Biophy. Meth., 28, 85-100, doi:10.1016/0165-022X(94)90023-X, 1994.

Barale, S., Vincent, L., Sauder, G., and Sbirrazzuoli, N.: Deconvolution of calorimeter response from electrical signals 
for extracting kinetic data, Thermochim. Acta, 615, 30-37, doi:10.1016/j.tca.2015.07.007, 2015.

Becker, L. and Gmehling, J.: Measurement of Heat Capacities for 12 Organic Substances by Tian-Calvet Calorimetry, J. Chem. Eng. Data, 46, 1638-1642, doi:10.1021/je010177o, 2001.

Brandenburg, A., Kita, J., Groß, A., and Moos, R.: Novel tubetype LTCC transducers with buried heaters and inner interdigitated electrodes as a platform for gas sensing at various high temperatures, Sensor. Actuat. B-Chem., 189, 80-88, doi:10.1016/j.snb.2012.12.119, 2013.

Calvet, É. and Prat, H.: Microcalorimétrie: Applications physicochimiques et biologiques, Masson, Paris, 1956.

Gongora-Rubio, M., Espinoza-Vallejos, P., Sola-Laguna, L., and Santiago-Avilés, J.: Overview of low temperature co-fired ceramics tape technology for meso-system technology (MsST), Sensor. Actuat. A-Phys., 89, 222-241, doi:10.1016/S09244247(00)00554-9, 2001.

Gotoh, M., Hill, K. D., and Murdock, E. G.: A gold/platinum thermocouple reference table, Rev. Sci. Instrum., 62, 2778, doi:10.1063/1.1142213, 1991.

Handy, B. E., Sharma, S. B., Spiewak, B. E., and Dumesic, J. A.: A Tian-Calvet heat-flux microcalorimeter for measurement of differential heats of adsorption, Meas. Sci. Technol., 4, 13501356, doi:10.1088/0957-0233/4/12/007, 1993.

Höhne, G., Hemminger, W., and Flammersheim, H.-J.: Differential scanning calorimetry: An introduction for practitioners, 2nd ed., Springer, Berlin, New York, XII, 298, 2003.

Imanaka, Y.: Multilayered low temperature cofired ceramics (LTCC) technology, Springer, New York, 2005.

Inaba, H., Takahashi, S., Mima, T., and Naito, K.: A high temperature Tian-Calvet type calorimeter and an analysis of the baseline fluctuation, J. Chem. Thermodynam., 16, 573-582, doi:10.1016/0021-9614(84)90009-0, 1984.

Jurków, D., Maeder, T., Dąbrowski, A., Zarnik, M. S., Belavič, D., Bartsch, H., and Müller, J.: Overview on low temperature cofired ceramic sensors, Sensor. Actuat. A-Phys., 233, 125-146, doi:10.1016/j.sna.2015.05.023, 2015.

Kita, J. and Moos, R.: Development of LTCC-materials and their applications - an overview, Informacije MIDEM, 38, 219-224, 2008.

Kita, J., Rettig, F., Moos, R., Drue, K.-H., and Thust, H.: Hot Plate Gas Sensors-Are Ceramics Better?, Int. J. Appl. Ceram. Tec., 2, 383-389, doi:10.1111/j.1744-7402.2005.02037.x, 2005.

Kita, J., Engelbrecht, A., Schubert, F., Groß, A., Rettig, F., and Moos, R.: Some practical points to consider with respect to thermal conductivity and electrical resistivity of ceramic substrates for high-temperature gas sensors, Sensor. Actuat. A-Phys., 213, 541-546, doi:10.1016/j.snb.2015.01.041, 2015.

Kleppa, O. J.: Evolution and application of high-temperature reaction calorimetry at the University of Chicago from 1952 to 2000, J. Alloy. Compd., 321, 153-163, doi:10.1016/S09258388(01)00964-1, 2001.

Löblich, K.-R.: On the characteristics of the signal curves of heat flux calorimeters in studies of reaction kinetics, Thermochim. Acta, 231, 7-20, doi:10.1016/0040-6031(94)80002-2, 1994.

Lyon, R. E. and Farris, R. J.: Analysis of heat-flux calorimeter signals, Thermochim. Acta, 161, 287-296, doi:10.1016/00406031(90)80310-U, 1990.
Markowski, P., Pinczakowski, W., Straszewski, L., and Dziedzic, A.: Thick-film thermoelectric microgenerators based on nickel-, silver- and PdAg-based compositions, 30th International Spring Seminar on Electronics Technology, Cluj-Napoca, Romania, 913 May 2007, 223-228, 2007.

Missal, W., Kita, J., Wappler, E., Gora, F., Kipka, A., Bartnitzek, T., Bechtold, F., Schabbel, D., Pawlowski, B., and Moos, R.: Miniaturized ceramic differential scanning calorimeter with integrated oven and crucible in LTCC technology, Sensor. Actuat. A-Phys., 172, 21-26, doi:10.1016/j.sna.2011.01.025, 2011.

Mudd, C., Berger, R. L., Hopkins, H. P., Friauf, W. S., and Gibson, C.: An optimized differential heat conduction solution microcalorimeter for thermal kinetic measurements, J. Biochem. Biophy. Meth., 6, 179-203, doi:10.1016/0165-022X(82)900422, 1982.

Razouk, R., Hay, B., and Himbert, M.: A new in situ electrical calibration system for high temperature Calvet calorimeters, Rev. Sci. Instrum., 84, 94903, doi:10.1063/1.4821876, 2013.

Saito, Y., Saito, K., and Atake, T.: Theoretical analysis of heat-flux differential scanning calorimetry based on a general model, Thermochim. Acta, 99, 299-307, doi:10.1016/0040-6031(86)852923, 1986.

Sarge, S. M., Gmelin, E., Höhne, G. W., Cammenga, H. K., Hemminger, W., and Eysel, W.: The caloric calibration of scanning calorimeters, Thermochim. Acta, 247, 129-168, doi:10.1016/0040-6031(94)80118-5, 1994.

Schönborn, K. H.: On the time lag between thermal event and measuring signal in a heat flux calorimeter, Thermochim. Acta, 69, 103-114, doi:10.1016/0040-6031(83)85069-2, 1983.

SETARAM Instrumentation: microSC - datasheet, available at: http://www.setaram.com/setaram-products/calorimetry/ microcalorimetry/microsc, 2015.

SETARAM Instrumentation: C80 - specifications, available at: http://www.setaram.com/setaram-products/calorimetry/ calorimetry-calorimetry/c80, 2016.

Tanaka, S.: Linear system theory of a heat conduction calorimeter, Thermochim. Acta, 141, 181-193, doi:10.1016/00406031(89)87053-4, 1989.

Topor, N. D. and Suponitskii, Y. L.: The High-temperature Microcalorimetry of Inorganic Substances, Russ. Chem. Rev., 53, 827-850, doi:10.1070/RC1984v053n09ABEH003127, 1984.

Touloukian, Y. S., Powell, R. W., He, J., and Klemens, P. G.: Thermal Conductivity: Metallic Elements and Alloys, Thermophysical Prosperties of Matter, 1, Springer US, Boston, MA, 1970.

Verein Deutscher Ingenieure (VDI): VDI-Wärmeatlas, 11th Edn., Springer Vieweg, Berlin, 2013.

Wiegärtner, S., Hagen, G., Kita, J., Reitmeier, W., Hien, M., Grass, P., and Moos, R.: Thermoelectric hydrocarbon sensor in thick-film technology for on-board-diagnostics of a diesel oxidation catalyst, Sensor. Actuat. B-Chem., 214, 234-240, doi:10.1016/j.snb.2015.02.083, 2015.

Zielenkiewicz, W.: How do the mathematical models of calorimeters really work?, Thermochim. Acta, 420, 23-27, doi:10.1016/j.tca.2003.09.037, 2004.

Zielenkiewicz, W., Margas, E., and Hatt, J.: Computerization of conduction calorimeters based on the N-body model, Thermochim. Acta, 88, 387-390, doi:10.1016/0040-6031(85)854599, 1985 . 